\title{
A New Electrocardiographic Criterium to Estimate a Septal or Lateral Location of a Right-Sided Accessory Pathway
}

José Nunes de Alencar Neto ${ }^{1, *}$, Rogério Gomes de Almeida Neto , Claudio Cirenza1, Angelo Amato Vincenzo de Paola1

ORCID IDS

Alencar Neto JN (1D https://orcid.org/0000-0002-3835-6067

Cirenza C (10 https://orcid.org/0000-0001-7290-9158

\begin{abstract}
There are algorithms to estimate the location of an accessory pathway (AP), but they tend to present low accuracy in right-sided APs. This paper presents a new electrocardiographic criterium to estimate the location of a right-sided AP. Rest ECGs from patients with manifest preexcitation of right-sided APs were evaluated and the SV2/RV3 ratio was calculated, considering values $<1$ for lateral (anterior or posterior) and $\geq 1$ for septal (anterior or posterior) APs. This ratio was compared with other signs already described in literature. In 175 consecutive patients, 60 met the inclusion criteria. For APs located in superior portions of the tricuspid ring, the SV2/RV3 ratio < 1 was 80\% accurate for anteroseptal location (specificity: 75\%), where His electrograms were recorded. For APs located in inferior portions of the tricuspid ring a SV2/RV3 < 1 was 82,86\% accurate for mid and posteroseptal location (specificity: 95.38\%). This work reports a new and simple criterium that can accurately distinguish right-sided lateral and septal APs with good specificity: SV2/RV3 ratio.
\end{abstract}

KEYWORDS: Cardiac arrhythmia; Electrocardiography; Accessory atrioventricular pathway. 


\section{INTRODUCTION}

In the general population, the prevalence of Wolff-Parkinson-White syndrome (WPW) is estimated to be 1-3 per 1000 individuals ${ }^{1-3}$. The syndrome is defined as the association of a short PR interval, a delta wave, and the occurrence of atrioventricular reentrant tachycardias (AVRTs) with retrograde conduction (more common) or anterograde by the nondecremental rapid conduction accessory pathway (AP) $)^{4,5}$.

Ablation of the accessory pathways is the treatment of choice in cases of APs with symptomatic and recurrent occurrences of AVRT or when there is high risk. In the periprocedural planning, the electrocardiographic analysis of the characteristics of the ventricular preexcitation is crucial because it guides the electrophysiologists to affirm if this accessory pathway is located in the tricuspid or mitral ring, if it is located next to some noble structure for the cardiac conduction system, more superiorly or more inferiorly, etc.

The literature shows several algorithms and flow charts ${ }^{6-9}$. Their accuracy for distinguishing mitral and tricuspid rings AP is high, and this allows electrophysiologists to plan a transseptal or retroaortic approach. However, in tricuspid ring APs, these algorithms tend to fail in determining the exact location. A peculiarly dangerous situation is an AP located in the anteroseptal position, where the His bundle is located. A radiofrequency application in this area is accompanied by a risk of complete heart block.

This study aimed to present two electrocardiographic criteria to accurately define the location of an accessory pathway in the tricuspid ring and compare it with other criterium already established in the literature.

\section{MATERIAL AND METHODS}

This work reviewed the procedural report and records and fluoroscopic images of all patients who underwent ablation of an AP at our institution between 2012 and 2018. Patients > 18 years with manifest preexcitation (QRS > $100 \mathrm{~ms}$ with evident delta wave) who underwent a successful ablation of a single AP located in the anteroseptal (para-Hisian), anterolateral, lateral, posterolateral, posterior, posteroseptal, and midseptal tricuspid ring were included. Particularly for para-Hisian and midseptal locations, the authors included patients whose AP was not successfully ablated because it is an infrequently encountered location, and radiofrequency was not always delivered.

The classification of APs based on the location was made in the left anterior oblique (LAO) view following these rules that are routinely employed in our service for right-sided pathways: (1) para-Hisian or anteroseptal at the place where the catheter records a satisfactory His bundle potential; (2) midseptal as the position between the catheter recording His bundle potential and that marking the superior border of the coronary sinus ostium, that is, between the 2 o'clock and 4 o'clock position; (3) right posteroseptal from the superior border of the coronary sinus ostium to the position around the 5 o'clock position; (4) the position is right posterior and posterolateral between the 5 o'clock position in the tricuspid annulus and a position that is exactly contralateral to the coronary sinus ostium, that is, the 8 o'clock position; (5) laterally and anterolaterally from this point of the tricuspid annulus in the right ventricular free wall to the point where anteroseptal pathways begins (Fig. 1).

Electrocardiograms (ECG) were recorded before procedure without pacing and were performed by routine in $25 \mathrm{~mm} / \mathrm{s}$ and $10 \mathrm{mV} / \mathrm{mm}$ with standardized positioning of the limb and precordial leads without pacing. Electrodes were positioned by the nurses, who receive constant training on electrode placement. The ECGs were analyzed specifically to determine if they were septal or lateral. The findings were compared with classic findings and algorithms already present in literature.

Based on the routine and previous signs already described, the authors proposed to test four criteria that could lead to a better accuracy. The first is the presence of any $\mathrm{r}$ wave in $\mathrm{V} 1$ (Fig. 2); the second is the precordial $\mathrm{R}$ wave transition occurring in V2 or V3; the third is the ratio between the $\mathrm{S}$ wave amplitude in V2 and the $\mathrm{R}$ wave amplitude in V3 (SV2/RV3 ratio) (Fig. 3); and the fourth, used only with accessory pathways located in inferior tricuspid portions, is the presence of an $\mathrm{R}$ 
wave amplitude in $\mathrm{V} 2 \geq \mathrm{R}$ wave amplitude in $\mathrm{V} 3$ (Fig. 4). Two comparisons were made: (1) para-Hisian vs. anterolateral and lateral AP; (2) right midseptal and posteroseptal vs. posterior and posterolateral AP. The results were analyzed for statistical significance by using the Fisher exact test $(<0.05$ was considered significant) followed by determination of its profiles of sensitivity (Se), specificity (Sp), positive predictive value (PPV), negative predictive value (NPV), and accuracy.

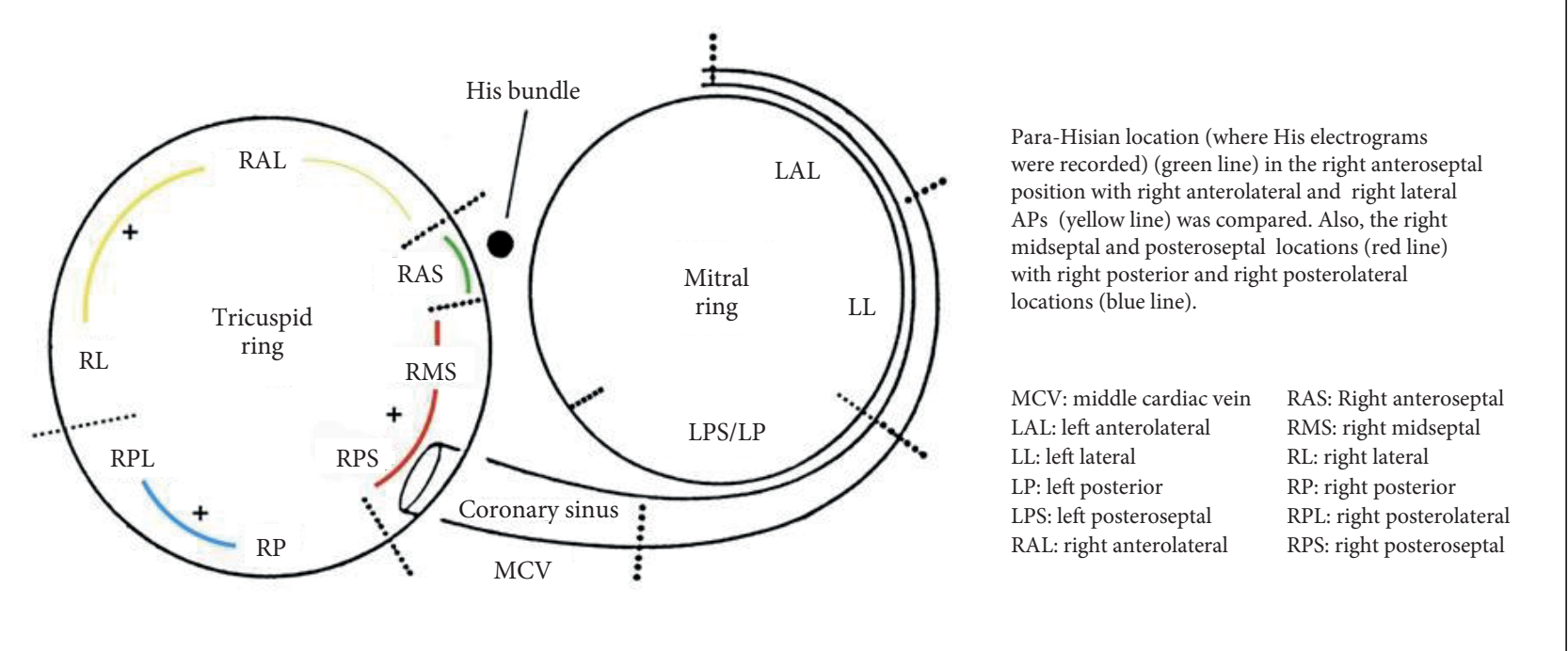

Figure 1. Detailed separation between AP in tricuspid and mitral rings used in this research.

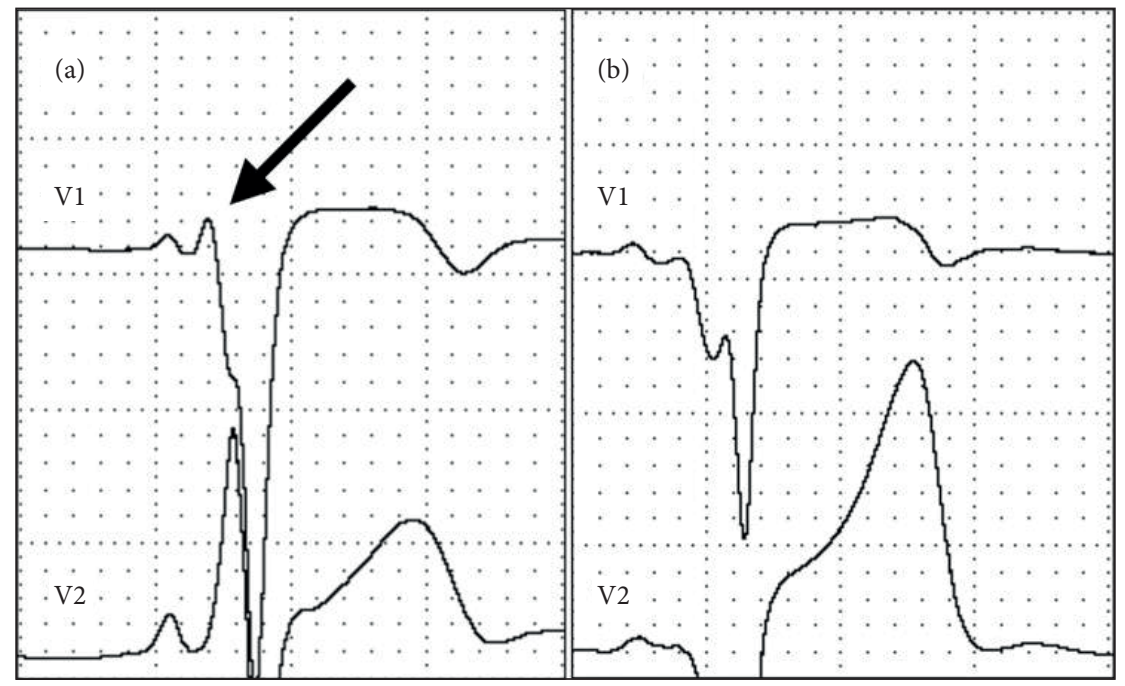

Figure 2. (a) An r wave of any duration in V1; (b) Absence of $r$ wave in V1. This sign favors lateral against septal APs according to Arruda's algorithm.

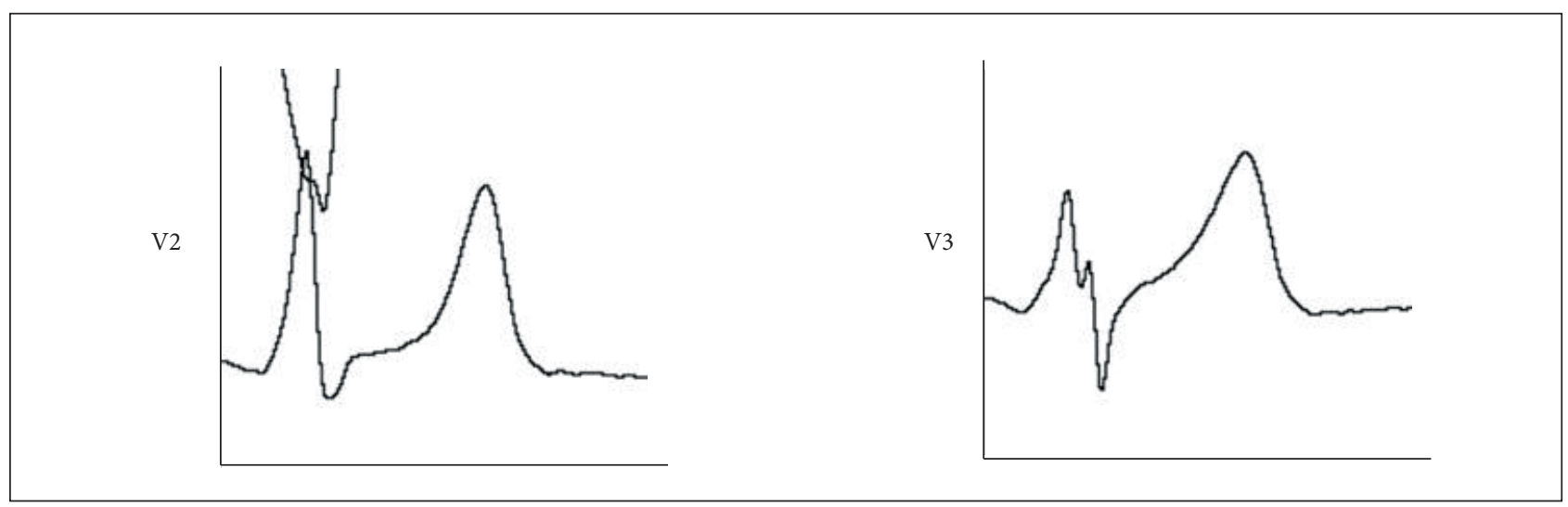

Figure 3. R wave in V2 with greater amplitude than R wave in V3. This sign favors lateral APs against septal APs. 


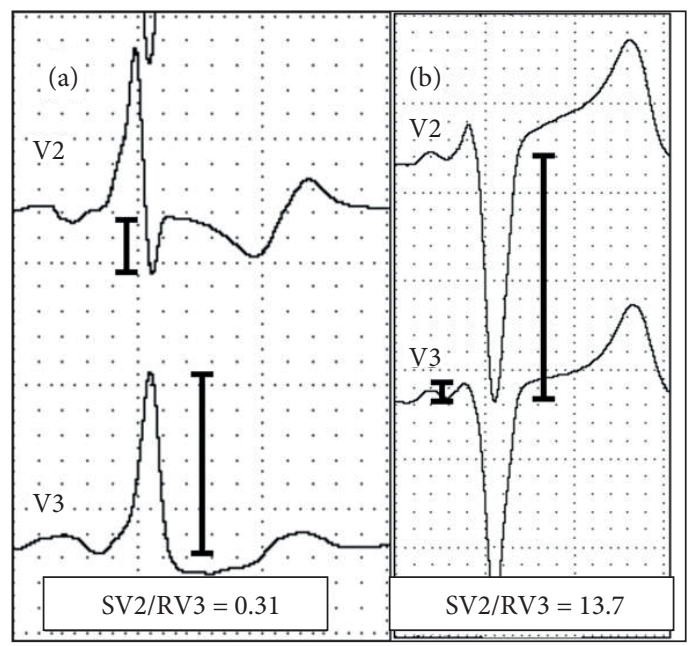

Figure 4. SV2/RV3 ratio measured by the division between $\mathrm{S}$ wave amplitude in V2 and the R wave amplitude in V3.

\section{RESULTS}

The procedures of 175 patients with manifest preexcitation who underwent ablation in the service were analyzed. From the total, 60 patients had APs in the locations prespecified for this study: 8 were right lateral or anterolateral; 17 with para-Hisian AP; 24 with right posteroseptal or mid-septal, and 11 with right posterior or posterolateral AP.

\section{Lateral/anterolateral versus para-Hisian APs}

To discriminate between anterolateral and lateral APs in detriment with para-Hisian location, the following signs were tested: the presence of an $r$ wave of any duration in V1. This sign was found in 8/8 of anterolateral and lateral APs and was absent in 7/17 of para-Hisian APs ( $p=0.0573$, Se: 100\%, Sp: 41.18\%, PPV: 44.44\%, NPV: 100\%, accuracy: $80 \%)$. The early transition of the $\mathrm{R}$ wave in precordial leads, that is, ( $\mathrm{R}>\mathrm{S}$ ) in V2 or V3 was also tested and showed $80 \%$ accuracy with a high sensitivity but low specificity profile (Se: 94,12\%, Sp: 50\%, PPV: 80\%, NPV: 80\%) for detection of para-Hisian APs (Table 1).

Table 1. Test profiles for distinguishing antero-septal APs (para-Hisian) from anterior and anterolateral.

\begin{tabular}{cccc}
\hline Sign/criterium & $\begin{array}{c}\text { R wave of any duration in V1 } \\
\text { ("Arruda" sign, favors lateral APs) }\end{array}$ & $\begin{array}{c}\text { R wave transition in V2 or V3 } \\
\text { (favors septal APs) }\end{array}$ & $\begin{array}{c}\text { SV2/RV3 ratio < 1 } \\
\text { (favors septal APs) }\end{array}$ \\
\hline Sensitivity & $100.00 \%$ & $94.12 \%$ & $82.35 \%$ \\
Specificity & $41.18 \%$ & $50.00 \%$ & $75.00 \%$ \\
PPV & $44.40 \%$ & $80.00 \%$ & $87.50 \%$ \\
NPV & $100.00 \%$ & $80.00 \%$ & $66.67 \%$ \\
Accuracy & $60.00 \%$ & $80.00 \%$ & $80.00 \%$ \\
\hline
\end{tabular}

AP = accessory pathways, NPV = negative predictive value, $P P V=$ positive predictive value

The SV2/RV3 ratio cut-off was $\geq 1$ for lateral/anterolateral and < 1 for para-Hisian APs ( $\mathrm{p}=0.0099$, accuracy: 80\%, Se: $82.35 \%$, Sp: 75\%, PPV: 87.5\%, NPV: 66.67\%).

\section{Posterior and posterolateral vs. midseptal and posteroseptal APs}

To discriminate between right midseptal or posteroseptal in detriment of posterior and posterolateral APs, the presence of an $r$ wave of any duration in V1 was tested as a sign of posterior/posterolateral AP (accuracy: 80\%, Se: 63.64\%, Sp: 87,5\%, PPV: 70\%, NPV: 84\%). An R wave transition in V2 or V3 had 77.78\% accuracy profile with low specificity for septal APs (Se 96\%, Sp 36.36\%, PPV 77.42\%, NPV: 80\%). 
The SV2/RV3 ratio cut-off value was $>1$ and $<1$ for posterior and posterolateral, and posteroseptal and midseptal, respectively. This ratio had an 82.86\% accuracy ( $\mathrm{p}=0.0017$, Se: 54.55\%, Sp: 95.38\%, PPV: 85.71\%, NPV: 82.14\%). The sign of an $\mathrm{R}$ wave with greater amplitude in V2 than in V3 had an 77.14\% accuracy (Se: 72.73\%, Sp: 79.17\%, PPV: 61.54\%, NPV: 86.46\%) (Table 2).

Table 2. Test profiles for distinguishing mid and posteroseptal APs from posterior and posterolateral.

\begin{tabular}{ccccc}
\hline Sign/criterium & $\begin{array}{c}\text { R wave of any duration } \\
\text { in V1 (favors posterior } \\
\text { and posterolateral APs) }\end{array}$ & $\begin{array}{c}\text { R wave transition in V2 } \\
\text { or V3 (favors mid and } \\
\text { posteroseptal APs) }\end{array}$ & $\begin{array}{c}\text { SV2/RV3 <1 (favors mid } \\
\text { and posteroseptal APs) }\end{array}$ & $\begin{array}{c}\text { R wave amplitude in } \\
\text { V2 V3 (favors mid and } \\
\text { posteroseptal APs) }\end{array}$ \\
\hline Sensitivity & $63.64 \%$ & $96.00 \%$ & $54.55 \%$ & $72.73 \%$ \\
Specificity & $87.50 \%$ & $36.36 \%$ & $95.83 \%$ & $79.17 \%$ \\
PPV & $70.00 \%$ & $77.42 \%$ & $85.71 \%$ & $61.54 \%$ \\
NPV & $84.00 \%$ & $80.00 \%$ & $82.14 \%$ & $86.46 \%$ \\
Accuracy & $80.00 \%$ & $77.78 \%$ & $82.86 \%$ & $77.14 \%$ \\
\hline
\end{tabular}

$\mathrm{AP}=$ accessory pathways, NPV = negative predictive value, $\mathrm{PPV}=$ positive predictive value .

\section{DISCUSSION}

This observational study proposes a new criterium to accurately determinate septal and lateral right-sided APs: the SV2/RV3 ratio, an adaptation of the "r wave precordial transition" proposed by Fitzpatrick et al., Milstein et al. and D'Avila et al. ${ }^{7-9}$. This ratio is calculated by dividing the $\mathrm{S}$ wave amplitude in $\mathrm{V} 2$ for the $\mathrm{R}$ wave amplitude in $\mathrm{V} 3$. If any of them is 0 , they should be considered 0.1 so the calculation would be feasible.

To discriminate between APs on the superior portion of the tricuspid ring (i.e., lateral and anterolateral vs. para-Hisian), the authors realized that the finding of an isolated $\mathrm{r}$ wave in V1 (from Arruda's algorithm) ${ }^{6}$ was not specific enough to exclude para-Hisian APs (60\% accuracy, Sp: 41.18\%) (Fig. 5). The SV2/RV3 ratio was applied and evidenced that a value of $<1$ is as accurate as $\mathrm{R}$ wave transition in V2 or V3 for para-Hisian APs ( $80 \%$ for both), but is more specific: (75\% vs. $50 \%$ in our sample) (Fig. 4 ).

In an AP ablation procedure, the electrophysiologist must be prepared to face complications. One of them is the unintended ablation of the His bundle, located in anteroseptal tricuspid ring. Delivering radiofrequency in this location leads to a great risk of developing complete heart block. This criterium must lead to a better preprocedural planning and wiser choices of catheters, for example, because ablation near the His bundle may be safer when performed with cryoablation technique ${ }^{10}$.

To discriminate between APs in the inferior portion of the tricuspid ring, the same signs already described were tested: the finding of any $r$ wave in V1 (Arruda's algorithm) seemed fairly accurate for posterior or posterolateral APs ( $87.5 \%$ specificity, with $80 \%$ accuracy) but low sensitivity (63.64\%). The SV2/RV3 ratio was also performed with a cut-off value of $\geq 1$ and $<1$ for posterolateral and posterior APs and septal APs, respectively. This test was $82.86 \%$ accurate, whereas the R wave transition in V2 or V3 for septal APs had 77.8\% accuracy. A new sign tested in our sample was the finding of an $\mathrm{R}$ wave of greater amplitude in V2 than in V3. It was accurate for mid and posteroseptal APs in detriment of posterior and posterolateral (Se: 72.73\%, Sp: 79.17\%, PPV: 61.54\%, NPV: 86.46\%) (Fig. 3).

Although the accuracy of the SV2/RV3 ratio is comparable to other signs already described, specially $\mathrm{R}$ wave transition in V2 or V3, this test had the best specificity profile (Tables 1 and 2). 


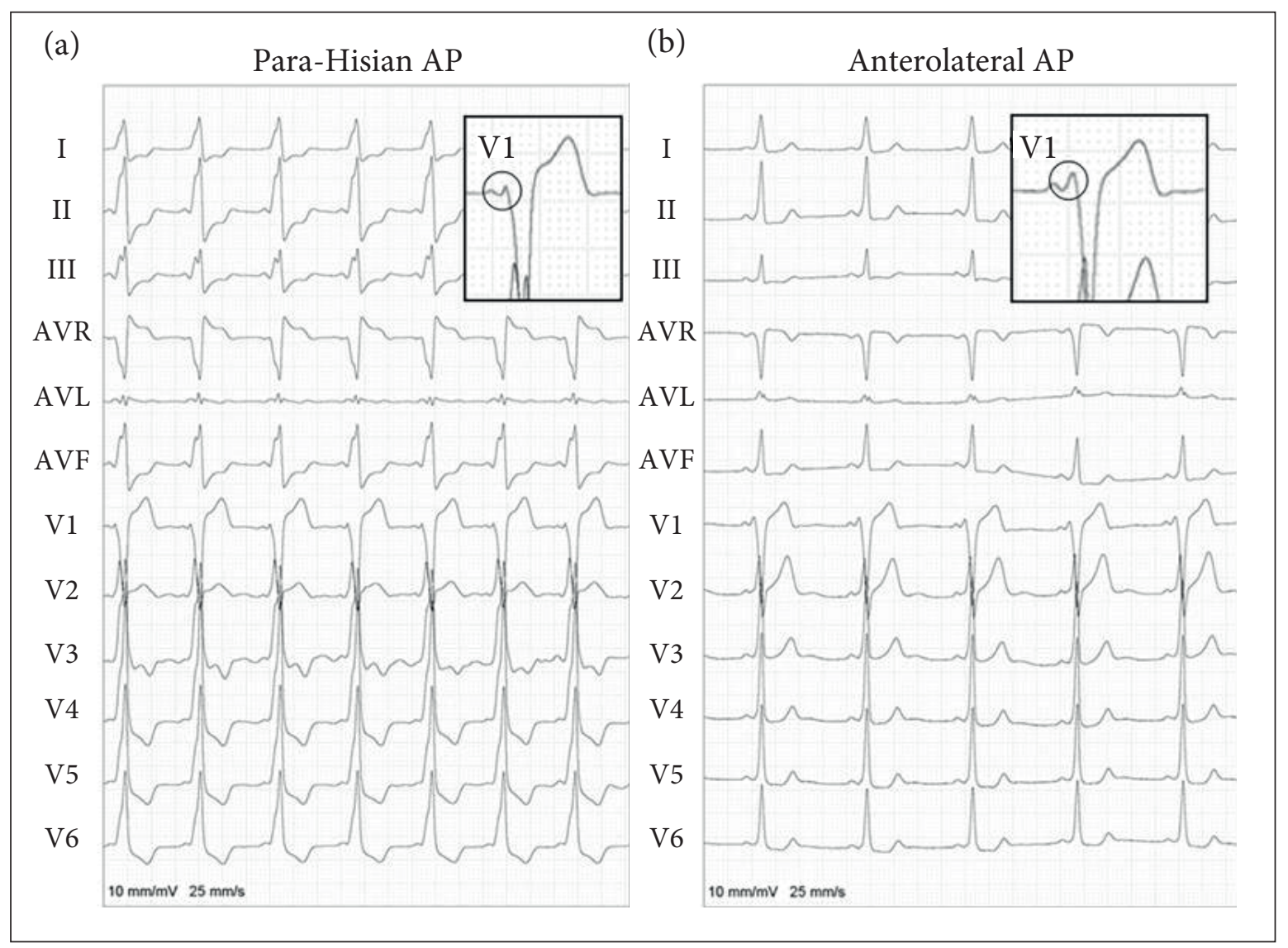

Figure 5. (a) Para-Hisian AP. Presence of a $r$ wave in V1 and R wave precordial transition in V2. (b) Anterolateral AP. Presence of a $r$ wave in V1 and R wave precordial transition in V2 are unable to distinguish lateral $x$ septal position. SV2/RV3 also fails. For these 2 ECGs, no signal proposed in literature would correctly differentiate them.

\section{Limitations}

Electroanatomic mapping was not performed in any of these cases because of financial limitations. Cryoablation was not performed either. Because cryoablation was not performed, many of the para-Hisian APs were not successfully ablated. The authors tried to exclude unsuccessful ablation procedures, but this was impossible in the para-Hisian location. The patients were maintained in the research to not diminish the sample size and lose statistical significance. However, in electrophysiology study, atrial and ventricular paces from different regions were routinely performed to unmask eventual bystander accessory pathways. The presence of two or more accessory pathways was an exclusion criterium.

As the goal of this research was to estimate, before procedural, the location of the accessory pathways, the electrocardiogram of these patients was acquired without pacing and with variable degrees of preexcitation. Thus, variations in the conduction of an accessory pathway and the AV node of patients may determine higher or lower preexcitation. Although this may be considered as a study limitation, this reflects exactly the daily practice of the electrophysiologist.

This was a single center retrospective study and therefore lacks predefined data collection techniques. Digital images were not always saved for review, and the location was based on the final procedure report. However, patients were excluded if anatomical description was lacking in the final report.

Finally, middle cardiac veins APs were not analyzed in this study. 


\section{CONCLUSION}

This work reports a new and simple criterium that can accurately distinguish right-sided para-Hisian from anterolateral and lateral AP and posteroseptal from posterior and posterolateral APs. Although the accuracy of the ratio is comparable to other signs described in literature, the specificity profile is better than the others. This criterium tends to facilitate periprocedural planning of the ablation approach.

\section{REFERENCES}

1. Krahn AD, Manfreda J, Tate RB, Mathewson FAL, Cuddy TE. The natural history of electrocardiographic preexcitation in men: the Manitoba Follow-up Study. Ann Intern Med. 1992;116(6):456-60. https://doi.org/10.7326/0003-4819-116-6-456

2. Hiss RG, Lamb LE. Electrocardiographic findings in 122,043 individuals. Circulation. 1962;25(6):947-61. https://doi.org/10.1161/01. CIR.25.6.947

3. Cohen MI, Triedman JK, Cannon BC, Davis AM, Drago F, JanousekJ, et al. PACES/HRS expert consensus statement on the management of the asymptomatic young patient with a Wolff-Parkinson-White (WPW, ventricular preexcitation) electrocardiographic pattern: Developed in partnership between the Pediatric and Congenital Electrophysiology Society (PACES) and the Heart Rhythm Society (HRS). Endorsed by the governing bodies of PACES, HRS, the American College of Cardiology Foundation (ACCF), the American Heart Association (AHA), the American Academy of Pediatrics (AAP), and the Canadian Heart Rhythm Society (CHRS). Hear Rhythm. 2012;9(6):1006-24. https://doi.org/10.1016/j.hrthm.2012.03.050

4. Guize L, Soria R, Chaouat JC, Chrétien JM, Houe D, Le Heuzey JY. Prévalence et évolution du syndrome de Wolff-Parkinson-White dans une population de 138048 sujets [Prevalence and course of Wolf-Parkinson-White syndrome in a population of 138,048 subjects]. Ann Med Interne (Paris). 1985;136(6):474-8. French.

5. Wolff L, Parkinson J, White PD. Bundle-branch block with short P-R interval in healthy young people prone to paroxysmal tachycardia. Am Heart J. 1930;5(6):685-704. https://doi.org/10.1016/S0002-8703(30)90086-5

6. Arruda MS, McClelland JH, Wang X, Beckman KJ, Widman LE, Gonzalez MD, et al. Development and validation of an ECG algorithm for identifying accessory pathway ablation site in Wolff-Parkinson-White syndrome. J Cardiovasc Electrophysiol. 1998;9(1):2-12. https:// doi.org/10.1111/j.1540-8167.1998.tb00861.x

7. Fitzpatrick AP, Gonzales RP, Lesh MD, Odin GW, Lee RJ, Scheinman MM. New algorithm for the localization of accessory atrioventricular connections using a baseline electrocardiogram. J Am Coll Cardiol. 1994;23(1):107-16. https://doi.org/10.1016/07351097(94)90508-8

8. Milstein S, Sharma AD, Guiraudon GM, Klein GJ. An algorithm for the electrocardiographic localization of accessory pathways in the Wolff-Parkinson-White syndrome. Pacing Clin Electrophysiol. 1987;10(3):555-63. https://doi.org/10.1111/j.1540-8159.1987. tb04520.x

9. D'Avila A, Brugada J, Skeberis V, Andries E, Sosa E, Brugada P. A fast and reliable algorithm to localize accessory pathways based on the polarity of the QRS complex on the surface ECG during sinus rhythm. Pacing Clin Electrophysiol. 1995;18(9):1615-27. https:// doi.org/10.1111/j.1540-8159.1995.tb06983.x

10. Gaita F, Riccardi R, Hocini M, Haissaguerre M, Giustetto C, Jais P, et al. Safety and efficacy of cryoablation of accessory pathways adjacent to the normal conduction system. J Cardiovasc Electrophysiol. 2003;14(8):825-9. https://doi.org/10.1046/j.15408167.2003.03076.x 